Preface

\title{
Obstetrics and Gynecology: A Specialty at the Forefront of Care for LGBTQ Patients
}

\author{
Iris L. Romero, MD, MS ${ }^{1}$ \\ ${ }^{1}$ Department of Obstetrics and Gynecology, University of Chicago, \\ Chicago, Illinois
}

Semin Reprod Med 2017;35:395-396

It is not our differences that divide us. It is our inability to recognize, accept, and celebrate those differences.

-Audre Lorde

In the relatively short tenure of our professional careers, there have been significant gains in the protection of civil rights for lesbian, gay, bisexual, transgender, and queer (LGBTQ) individuals. The culmination of considerable effort was the U.S. Supreme Court's ruling (Obergefell $v$. Hodges in 2015 ) that gay marriage is a constitutional right that must be recognized by all states. Mirroring the progress made on the legal front, the health care system has also made considerable strides in caring for this patient population. The days when homosexuality was classified as a mental disorder, for example, are long past. However, health care in general, and obstetrics and gynecology specifically, has not kept pace with the needs of this community. Fundamentally, the gains in legal protection made for LGBTQ individuals and their families will be insufficient if the health care system does not honor our commitment to the health and well-being of all of our patients. As obstetricians, gynecologists, and midwives, we are uniquely positioned at the forefront of this journey to health care equality.

In this issue of Seminars in Reproductive Medicine (SRM), our aim is to arm healthcare providers with current data and best practices so they will be well positioned to meet the health care needs of their LGBTQ patients. To this end, we move beyond the basics of LGBTQ health and focus on topics that are contemporary priorities for LBGTQ patients. National experts were recruited to contribute articles in designated topic areas. The authors were not only asked to give a rigorous review of the subject matter but also to provide practical clinical applications for their findings. The cornerstone of this issue of SRM is a call to action to establish culturally competent clinical practices using the roadmap outlined by Cook et al in Establishing Effective Health Care Partnerships with Sexual and Gender Minority Patients: Recommendations for Obstetrician Gynecologists.

Understanding that the gains in legal protection have translated into an increase in LGBTQ individuals pursuing parenthood, the first section of the issue focuses on the provision of reproductive care. First, in the article entitled Family Building by Same-Sex Male Couples Via Gestational Surrogacy, H. Kim reviews best practices for infertility treatment for gay parents, including a review of federal regulations. Complimenting this article, and on the cutting edge of assisted reproductive technology, Getrajdman et al, in an article entitled Co-IVF for Same-Sex Female Couples, provides a comprehensive review of an approach which involves oocytes retrieval from one partner and the transfer of the fertilized embryo to the other partner, who will carry the pregnancy. Finally, recognizing the pivotal role obstetricians and midwives play in the pathway to parenthood, the article Lesbian Pregnancy: Care and Considerations by Bushe et al provides culturally sensitive guidance for caring for lesbian mothers from preconception through postpartum care, including specific considerations for the nonbiologic mother.

Next, this issue of SRM focuses on an area where our specialty is perhaps the least proficient-transgender health. First, in Preventive Health for Transgender Men and Women, Imborek et al provide busy clinicians with a comprehensive and practical approach to long-term, preventive-health-focused, primary care for transgender patients. Next, in an article focused on breast cancer-Breast Cancer Screening, Management, and Review of Case Study Literature in Transgender Populations-Deutsch et al review the literature and provide clinical recommendations. Finally, to prepare gynecologists for the role they may play as transgender women transition to their affirmed gender, C.A. Unger
Address for correspondence Iris L. Romero, MD, MS, Department of Obstetrics and Gynecology, University of Chicago, 5841 S Maryland Avenue, MC2050, Chicago, IL 60637 (e-mail: iromero@bsd.uchicago. edu).
Issue Theme Obstetrics and Gynecology: A Specialty at the Forefront of Care for LGBTQ Patients; Guest Editor, Iris L. Romero, MD, MS
Copyright (c) 2017 by Thieme Medical Publishers, Inc., 333 Seventh Avenue, New York, NY 10001, USA. Tel: +1(212) 584-4662.
DOI https://doi.org/ 10.1055/s-0037-1607044. ISSN 1526-8004. 
reviews guidelines for surgical and cross-sex hormone therapy in Update on Gender-Affirming Treatment for the Transgender Woman.

The final topic in this issue of SRM ventures into uncharted waters. First, in the article entitled Sexual Dysfunction in Lesbian Women: A Systematic Review of the Literature, Sobecki-Rausch et al provide the most comprehensive review to date on sexual dysfunction in lesbians. Second, in the highly novel article, Family Planning for Sexual Minority Women, Stoffel et al describe a surprisingly high rate of unintended pregnancy and unmet family planning needs in this patient population. These two articles, along with the others outlined earlier, highlight the invisibility of LGBTQ patients in many health care initiatives and research priorities. Work in these arenas has been significantly constrained by insufficient data collection. Therefore, an important first step would be the inclusion of gender and sexual identity in federally funded surveys, as was recommended by the Institute of Medicine in the 2011 report "The Health of Lesbian, Gay, Bisexual, and Transgender People: Building a Foundation for Better Understanding."1 Only when all important research endeavors include LGBTQ individuals will this patient population begin to reap the benefits of evidencebased medicine.

While significant work remains, this issue of SRM is a meaningful step in the right direction. As the guest editor, I would like to thank all of the authors for contributing their time and expertise to help our specialty in our goal to provide comprehensive, culturally sensitive, and equitable care to all of our patients. I also applaud Seminars in Reproductive Medicine for undertaking such an important topic and the journal editors for their demonstrated commitment to advocacy and allyship for LGBTQ patients. As other journals, professional societies, and research priorities follow SRM's example, we will truly begin to, as Audre Lorde said, recognize, accept, and celebrate the differences among us.

\section{Reference}

1 Institute of Medicine Committee on Lesbian, Gay, Bisexual, and Transgender Health Issues and Research Gaps and Opportunities. The National Academies Collection: Reports funded by National Institutes of Health. The Health of Lesbian, Gay, Bisexual, and Transgender People: Building a Foundation for Better Understanding. Washington, DC: National Academies Press (US); National Academy of Sciences; 2011 